\title{
Metal-silicate mixing by large Earth-forming impacts
}

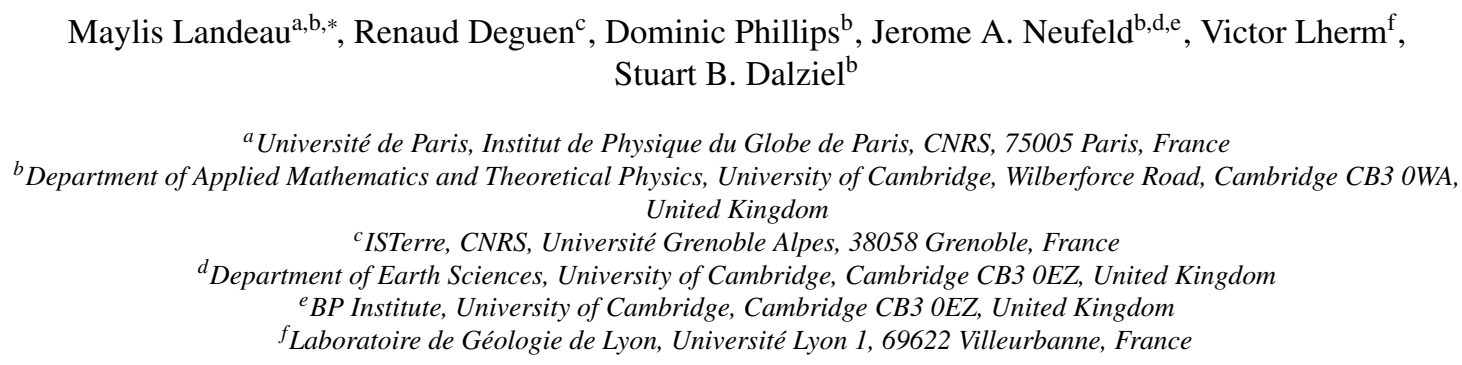

\section{Abstract}

Geochemical and isotopic observations constrain the timing, temperature and pressure of Earth's forma- 


\section{Introduction}

The Earth formed 4.6 billion years ago (Patterson et al., 1955) by successive planetary collisions. During this process, the metallic core and the silicate mantle differentiated and acquired their initial temperature and composition. These initial conditions shaped the present-day structure and dynamics of our planet. They determined the beginning of the geodynamo (Badro et al., 2018), the initiation of plate tectonics (Bercovici and Ricard, 2014) and the nucleation of the inner core (Olson et al., 2015).

Geochemical observations tell us about Earth's differentiation. Isotopic ratios and extinct radioactivity indicate that the core formed in about $30 \mathrm{Myr}$ (Kleine et al., 2002). During differentiation, chemical species partition into metal and silicates depending on pressure and temperature. Mantle abundances in siderophile elements suggest the separation of metal and silicates at a high temperature of $3000-3500 \mathrm{~K}$ and depths of 1000 - 1500 km (Siebert et al., 2012; Fischer et al., 2015).

However, these estimates all assume that metal was in thermodynamic equilibrium with the entire mantle when it segregated into the core. Recent models relax this assumption, allowing for equilibration of only fractions of the core and mantle. With these assumptions, the duration of core formation, deduced from isotopic ratios, becomes indeterminate, varying from 30 Myr to 100 Myr or more (Rudge et al., 2010). Similarly, the equilibrium pressure deduced from siderophile abundances extends to that of the core-mantle boundary when allowing for partial equilibration (Fischer et al., 2017). An accurate assessment of chemical transfers is therefore needed to fully interpret geochemical observations. These transfers depend on the efficiency of mixing between metal and silicates, and hence on the fluid dynamics of Earth's differentiation (Rubie et al., 2003).

Models of planetary formation suggest that much of Earth's mass accreted by collisions between planetary embryos composed of a metallic core and a silicate mantle (Chambers, 2004; Ricard et al., 2009). Each impact produced shock-waves that melted the colliding embryos, releasing the liquid core of the impactor in a fully-molten magma ocean (Fig.1a) (Tonks and Melosh, 1993; Nakajima et al., 2020). The impactor core sank into this ocean and merged with the core of the target embryo. During this process, the dynamics were highly turbulent: inertia was large compared to surface tension and viscous forces (Dahl and Stevenson, 2010; Deguen et al., 2011). Turbulence mixed the impactor core and the magma ocean down to small-scales, increasing the rate of chemical equilibration.

The first physical estimates of this mixing come from theoretical and numerical calculations (Rubie et al., 2003; Ichikawa et al., 2010; Ulvrová et al., 2011; Samuel, 2012; Qaddah et al., 2019; Maas et al., 2021). These suggest that, following a collision, the impactor core broke up into centimetric drops, forming a rain of metal in the magma ocean. Laboratory experiments on the fragmentation of a liquid volume find different dynamics (Landeau et al., 2014; Deguen et al., 2014). They suggest that, instead of forming a

${ }^{*}$ Corresponding author

Email address: landeau@ipgp.fr (Maylis Landeau) 
rain, the impactor core fell in the ocean as a dense, coherent cloud that entrained silicates. This structure is called a turbulent thermal in fluid dynamics (Morton et al., 1956). In the thermal, the impactor metal was stretched and convolved with the entrained silicates (Lherm and Deguen, 2018). It eventually fragmented into millimetric drops at depths $4-8$ times the radius of the impactor core (Landeau et al., 2014). By fragmenting, the impactor metal increased its surface area, thus enhancing chemical transfers. However, the impactor core equilibrated only with the silicates entrained inside the thermal, i.e. only with a fraction of the mantle. From existing theories on thermals, Deguen et al. (2014) develop a predictive model for the volume of mixed silicates and the degree of equilibration. Wacheul and Le Bars (2018) recently validate these predictions in immiscible thermals.

However, this picture is still incomplete. These previous investigations all neglect the inertia of the impactor and the disruption caused by the impact. This is at odds with geological observations on terrestrial craters, which indicate that the impactor was strongly dispersed within the target. These observations are particularly clear for Meteor Crater (Arizona, USA), where we find fragments of the iron meteorite that formed the crater kilometres away from the impact point (Blau et al., 1973; Vdovykin, 1973).

These observations raise an important, still unresolved, question: During an Earth-forming collision, was the impactor core dispersed? If it was, metal-silicate equilibration was stronger than the estimates of Deguen et al. (2014). In this work, we use fluid dynamical experiments to quantify mixing by a large planetary impact and reassess metal-silicate equilibration.

\section{Modelling planetary impacts}

The most widely recognised method to investigate planetary impacts is numerical modelling (Canup, 2004; Ćuk and Stewart, 2012; Kendall and Melosh, 2016). Kendall and Melosh (2016) use high-resolution simulations to characterise the stretching of the impactor core. They observe that, on impact, the core spreads into a thin layer over the deforming crater floor. The crater then collapses and rises as a vertical jet, stretching the core to an even greater extent. However, while the simulations of Kendall and Melosh (2016) resolve scales down to $2.5 \mathrm{~km}$, the typical length scale for chemical equilibration is $1 \mathrm{~cm}$ (Rubie et al., 2003; Ulvrová et al., 2011).

Here, to model turbulent mixing down to such small scales, we use analogue laboratory experiments. We investigate liquid volumes falling in air and impacting into another miscible liquid (Fig. 1b). We quantify mixing between the released volume, representing the impactor, and the target, representing the magma ocean, as a function of the impact speed. Unlike impact simulations, these experiments are subsonic and cannot produce significant heating. In addition, our impactors are not differentiated into a core and a mantle. However, these experiments complement impact simulations by accurately constraining small-scale mixing, which is crucial in estimating metal-silicate equilibration.

Why would impacts between Newtonian liquids reproduce the dynamics of solid planetary impacts? 
Collisions onto a planetary embryo larger than $2000 \mathrm{~km}$ in diameter produce shock waves that melt both the impactor and target (Tonks and Melosh, 1993). In addition, the pressures produced by impactors $\gtrsim 100 \mathrm{~km}$ are much larger than the strength of the solid mantle. This is the so-called gravity regime (Melosh, 1989). In this regime, the impactor and target behave as fluids.

In planetary impacts, the impactor core and the magma ocean are immiscible. This immiscibility controls the size of the metal fragments (Deguen et al., 2011; Wacheul and Le Bars, 2018). Here, we do not investigate this size. Instead, we focus on the overall volume of silicates entrained with metal. During a planetary impact, inertial forces are much larger than interfacial forces between metal and silicates (Deguen et al., 2011). In this regime, the rate of entrainment is identical to that observed in miscible liquids (Landeau et al., 2014). Metal-silicate immiscibility therefore plays no role in the entrainment; hence, we study miscible impactor and target liquids here.

Still, in our experiments and planetary impacts, the target and impactor are immiscible with the air. The impact of millimetric drops at an air-liquid interface is a classical, yet active, topic in fluid dynamics (Worthington, 1908; Ray et al., 2015). During drop impacts, viscous dissipation and surface tension with air play an important role. In contrast, both effects are small compared to gravity and inertia during large planetary impacts. To reach this inertial regime, we conduct the first impact experiments with large decimetre-sized liquid impactors.

Here, we first introduce the experimental setup (section 3). After a qualitative description of the impact dynamics (section 4.1), we demonstrate that our experiments replicate the cratering process observed in previous subsonic impacts (section 4.2). We derive scaling laws for the mixing between the impactor and the target (section 4.3), which we explain by scaling arguments (section 4.4). Finally, we translate our scalings into improved estimates for the degree of equilibration (section 5.2).

\section{Methods}

\subsection{Experimental set-up}

Fig.1 shows the geophysical motivation and the experimental set-up. A volume of salt solution of density $\rho_{i}$ and radius $R$ impacts a target liquid of density $\rho_{t}$, representing the magma ocean. The target liquid is held in an acrylic tank, with a $76 \mathrm{~cm}$ square planform, filled with fresh water to a depth of $50 \mathrm{~cm}$. We vary the impact velocity $U$ by varying the release height of the impactor.

In this study, we take $R \gtrsim 3 \mathrm{~cm}$. We initially hold the impacting liquid in a latex balloon. We place a needle below the balloon, at less than $30 \mathrm{~cm}$ above the target surface. When we release the balloon, it falls onto the needle, which ruptures the latex membrane and releases a nearly spherical liquid volume.

We visualise the flow by back illumination through a diffusive screen. The light source, measuring 90 $\mathrm{cm} \times 120 \mathrm{~cm}$, is a panel of red LEDs driven by a stabilised DC power supply. In order to characterise fluid mixing, we dye the impacting liquid using blue food colouring at a volumetric concentration of $0.025 \%$. We 

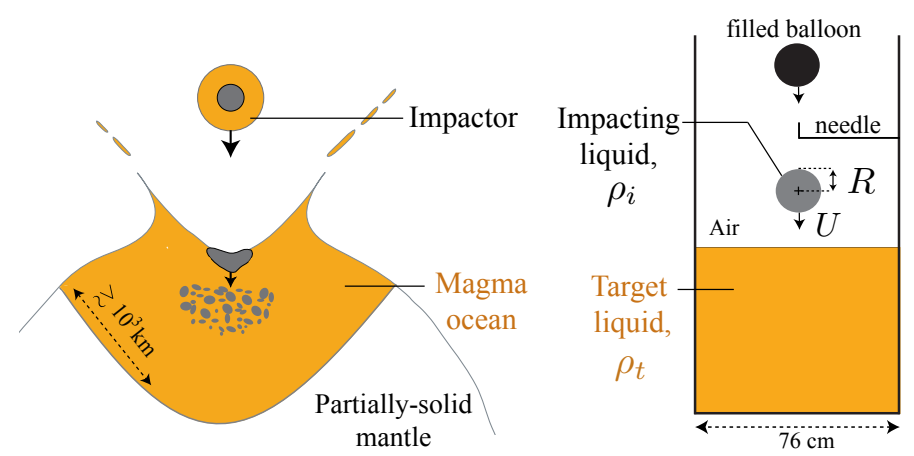

Figure 1: (a) Metal-silicate mixing by a planetary impact; the impactor core is in grey, the magma ocean in yellow and the partiallysolid mantle in white. In this sketch, the magma pool is generated by the impact (Melosh, 1990; Nakajima et al., 2020). Alternatively, the magma ocean can predate the impact; it then forms a layer of uniform thickness. (b) Experimental set-up (side view). A liquid impactor of radius $R$ and density $\rho_{i}$ impacts a target liquid of density $\rho_{t}<\rho_{i}$ at velocity $U$. The impacting and pool liquids are analogues for the impactor and the magma ocean, respectively.

also conduct a series of experiments with no dye to measure precisely the transient crater depth. The flow is recorded with a Photron SA1.1 monochrome high-speed camera with a resolution of $1024 \times 1024$ pixels. We use 1000 frames per second to image the impact stage (see section 4.1) and 125 frames per second for the slower buoyancy-induced thermal stage (as defined in section 4.1).

In order to vary the density of the impacting liquid, we use solutions of sodium chloride $(\mathrm{NaCl})$ at different concentrations to give densities between $\rho_{i}=998.66 \mathrm{~kg} \mathrm{~m}^{-3}$ and $1100.78 \mathrm{~kg} \mathrm{~m}^{-3}$. The target pool density is fixed at $\rho_{t}=998.66 \mathrm{~kg} \mathrm{~m}^{-3}$. For each experiment we weigh the impacting liquid and measure its density using an Anton Paar DMA 5000 density meter, from which we deduce the equivalent spherical radius $R$ of the impactor.

\subsection{Dimensionless numbers}

The following dimensionless numbers govern the dynamics in planetary impacts and in our experiments:

$$
\begin{aligned}
& F r=\frac{U^{2}}{g R}, \quad P=\frac{\rho_{i}-\rho_{t}}{\rho_{t}}, \\
& W e=\frac{\rho_{i} U^{2} R}{\sigma_{t}}, \quad \operatorname{Re}=\frac{U R}{v_{t}}, \quad M=\frac{U}{U_{\mathrm{s}}}, \quad \frac{v_{i}}{v_{t}}, \quad \frac{\sigma_{i}}{\sigma_{t}} .
\end{aligned}
$$

The impact Froude number Fr measures the importance of the impactor kinetic energy to its gravitational energy at impact, as discussed in supplementary section S1, where $g$ is the gravitational acceleration. The Weber number $W e$ and the Reynolds number Re measure the importance of the impactor kinetic energy to the interfacial energy and viscous dissipation, respectively. The ratio $P$ is the normalised excess density of the impactor. The Mach number $M$ is the ratio of the impact velocity $U$ to the speed of sound $U_{s}$, and $v_{i} / v_{t}$ and $\sigma_{i} / \sigma_{t}$ are the ratios of impactor to target kinematic viscosities and surface tensions with air, respectively. Supplementary Table 1 compares the values of these parameters in our experiments and in the 
large impacts of Earth's formation.

During a planetary impact, the Mach number is $M=1-10$. We cannot reach these supersonic conditions when releasing a liquid impactor in the laboratory. Instead, our experiments are in the limit of low Mach number where compressibility effects are negligible.

Despite their low Mach number, the experiments reported here are in the limit where the impactor kinetic energy is large compared with viscous dissipation and interfacial energies $\left(\operatorname{Re}>10^{3}\right.$ and $\left.W e>5 \times 10^{4}\right)$. Although these values are far from the values for planetary impacts (supplementary Table 1), they are among the highest reported for liquid impacts (Bisighini et al., 2010; Ray et al., 2015). This implies that in our experiments, and in planetary collisions, inertia and buoyancy forces govern the dynamics, and hence determine the mixing (Ellison and Turner, 1959; Landeau et al., 2014). The experimental results can therefore be described solely in terms of two dimensionless control parameters: the normalised excess density $P$ and the impact Froude number $F r$. The Froude numbers $F r$ in our experiments match those of planetary impactors with a radius $\gtrsim 100 \mathrm{~km}$ (supplementary Table 1$)$.

\subsection{Diagnostics}

In each experiment, we measure the size of the impactor $R$, and the impact velocity $U$, from which we construct the impact Froude number Fr. From each frame before the impactor reaches the target, we locate the $2 \mathrm{D}$ centroid of the impactor. We fit the position of this centroid as a function of time with a quadratic polynomial. From this fit, we compute the velocity $U$ at the time when the centroid intersects the surface of the target.

To quantify mixing between the dyed impactor and the target, we use the light-attenuation technique (Cenedese and Dalziel, 1998). As detailed in supplementary section S2, we measure the vertical position $z$ of the centre of mass of the dyed liquid and its characteristic volume $\tilde{V}$ normalised by the impactor volume. From this dimensionless volume $\tilde{V}$, we define the equivalent spherical radius of the dyed liquid $r=R \tilde{V}^{1 / 3}$. Uncertainties in $U, z$ and $\tilde{V}$ are typically on the order of $5 \%, 5 \%$, and $15 \%$, respectively. Uncertainties in $U$ mainly come from the retraction of the latex membrane that produces spurious liquid spray at the impactor surface. Uncertainties in $z$ and $\tilde{V}$ mainly come from light reflections and refractions by waves generated by the impact at the target surface.

\section{Results}

\subsection{Experimental observations}

In all our experiments, we observe two main stages. At early times, the impact process dominates the dynamics (Fig.2). At later times, buoyancy forces become important and the impactor sinks into the lighter target pool, forming the so-called turbulent thermal (Fig.3). Below, we describe these two stages. 

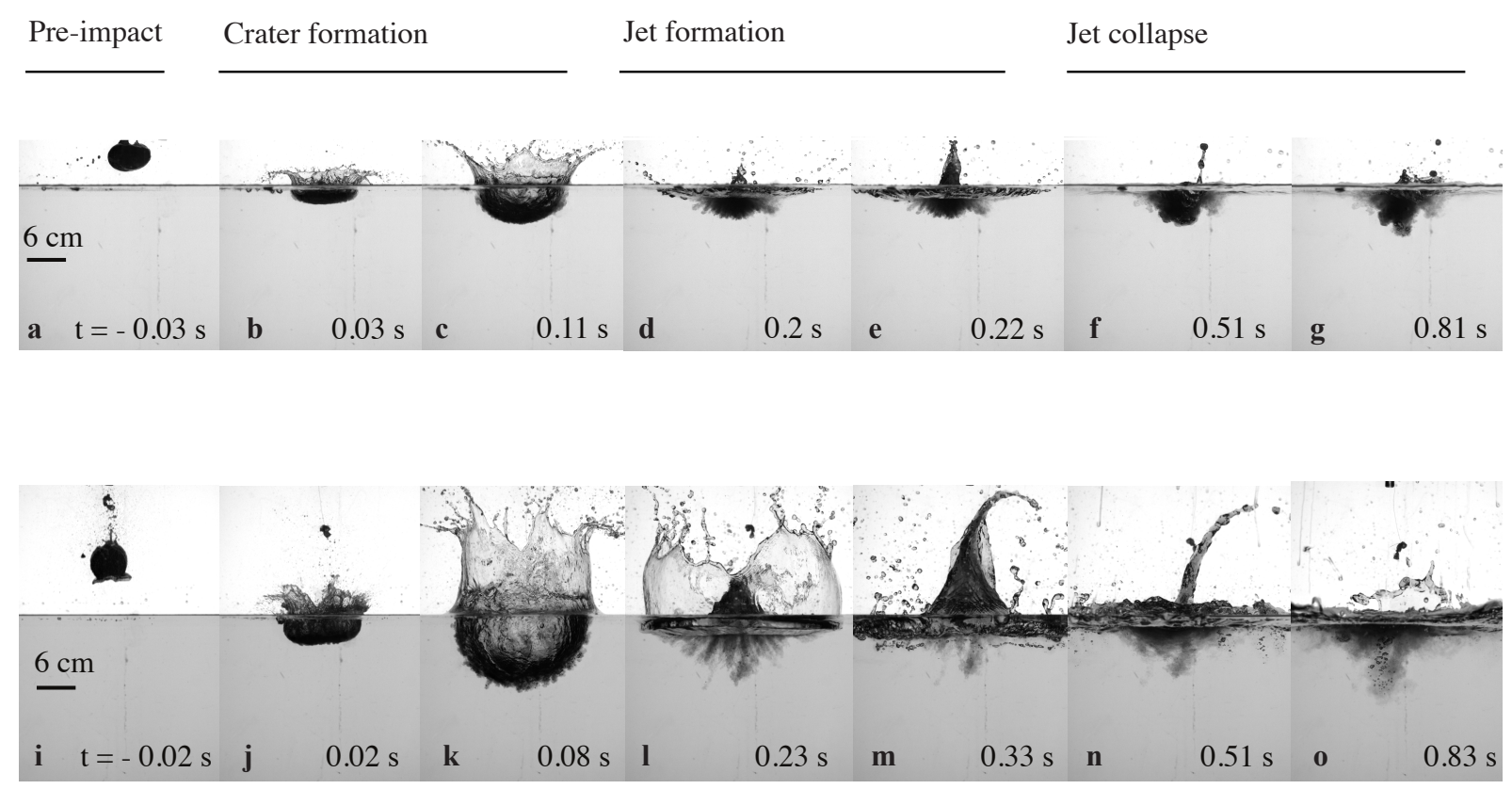

Figure 2: Early-time impact stage: impact cratering (b,c, and j,k), jet formation (d,e and 1,m), jet collapse (f,g and n,o). First line: Low impact Froude number, $F r \simeq 6, U \simeq 1.3 \mathrm{~m} \mathrm{~s}^{-1}$. Second line: High impact Froude number, $F r \simeq 100, U \simeq 5.4 \mathrm{~m} \mathrm{~s}^{-1}$. In both experiments $P \simeq 0.02$ and the impactor radius $R \simeq 3 \mathrm{~cm}$. The experiments in the first and second lines correspond to supplementary movies S1 and S2, respectively.

\subsubsection{Short-time impact stage}

The impact-dominated stage lasts about $1 \mathrm{~s}$ (Fig.2 and supplementary movies S1 and S2). We observe three successive processes in the impact stage.

The first process corresponds to the formation of a crater (Fig.2b,c and Fig.2j,k). The crater expands until its gravitational energy nearly matches the initial kinetic energy of the impactor (Pumphrey and Elmore, 1990; Ray et al., 2015); at this point the crater reaches its maximum depth (Fig.2c and 2k). During the formation of the crater, we observe mushroom-shaped instabilities at the interface between the impactor and the target (Fig.4). These instabilities induce some mixing between the two fluids. We find that these structures disappear in the absence of a density difference, i.e. for $P=0$. We can understand these instabilities by considering the acceleration history of the fluids.

At the moment of impact, the impactor and target fluids are impulsively decelerated and accelerated, respectively. This impulsive acceleration can trigger the growth of perturbations through an incompressible Richtmyer-Meshkov mechanism (Jacobs and Sheeley, 1996; Lund and Dalziel, 2014). This growth is then modified during the opening of the crater. The crater floor decelerates until it reaches its maximum depth (Fig.2c,k). This deceleration, together with the gravitational acceleration, drives a Rayleigh-Taylor instability at the interface between the dense impactor and the lighter pool (Rayleigh, 1883).

The second process in the impact stage is the collapse of the crater because of gravity and the formation of a jet (Fig.2de and Fig.2lm). This jet is similar to that observed in drop impacts (Ghabache et al., 2014).

The jet eventually stops rising, marking the end of jet formation and the beginning of the jet collapse 

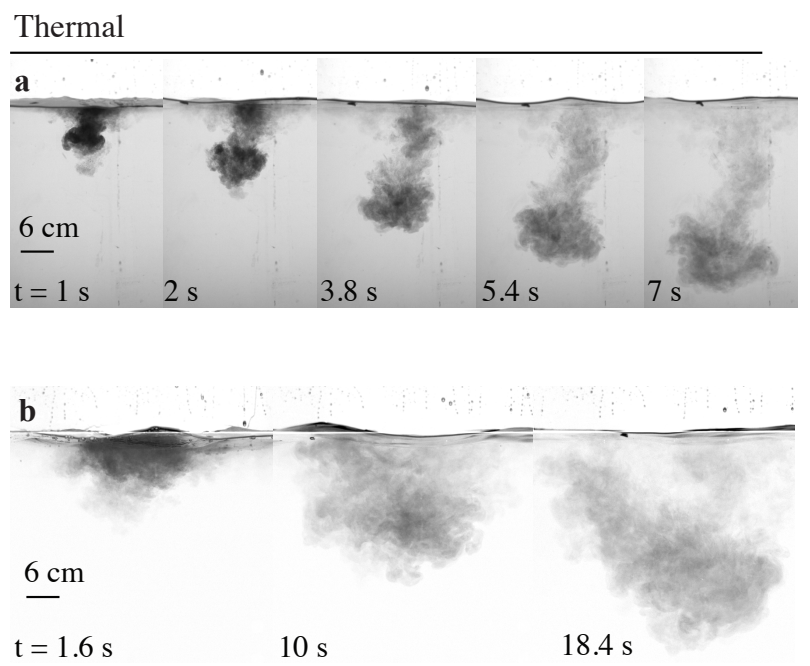

Figure 3: Late-time thermal stage following the impact shown in Fig.2. (a) Low impact Froude number, $F r \simeq 6, U \simeq 1.3 \mathrm{~ms}$. (b) High impact Froude number, $F r \simeq 100, U \simeq 5.4 \mathrm{~m} \mathrm{~s}^{-1}$. In both experiments $P \simeq 0.02$ and the impactor radius $R \simeq 3 \mathrm{~cm}$. The experiments in (a) and (b) correspond to supplementary movies S1 and S2, respectively.

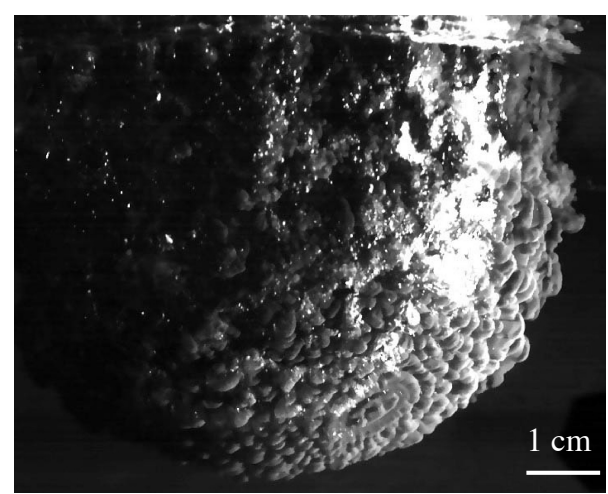

Figure 4: Mushroom-shaped structures observed during the crater opening process shown in Fig.2a,b,c and Fig.2i,j,k. In this experiment, $F r \simeq 20$ and $P \simeq 0.18$. To obtain this image we added fluorescein in the impacting liquid and we filmed the impact from below, illuminating it from the side with a halogen lamp.

process (Fig.2f and Fig.2n). The jet collapses under its own weight, accelerating the fluid it contains downwards and causing additional mixing between the impactor and the target.

The experiment shown in the first row of Fig.2 has $F r \simeq 6$ (supplementary movie S1), while that in the second row has $F r \simeq 100$ (supplementary movie S2). We observe that the maximum crater depth, the extent of the splash and the height of the secondary jet, all normalised by the impactor size, increase with increasing impact Froude number.

\subsubsection{Long-time thermal stage}

The denser impacting fluid eventually descends into the lighter target (Fig.3). This stage introduces mixing over a much longer timescale, resulting from the total buoyancy of the impacting liquid in the target. The impacting liquid forms a buoyant cloud that grows by entrainment of target liquid, the so-called thermal (Taylor, 1945; Batchelor, 1954; Morton et al., 1956; Scorer, 1957).

Recently, several investigations used turbulent thermals to model core formation (Deguen et al., 2011, 2014; Landeau et al., 2014; Wacheul and Le Bars, 2018). However, these studies lack an initial impact stage: 
the analogue fluid for the impactor core was initially immersed in the ambient and at rest. It is therefore noteworthy that we recover a turbulent thermal in our impact experiments. Still, including the impact stage is crucial: the extent of the thermal, and hence the mixing, is larger with $F r \simeq 100$ (Fig.3b) than with $F r \simeq 6$ (Fig.3a). The Froude number also affects the initial shape of the thermal, which is spherical with $F r \simeq 6$ (Fig.3a) but closer to a hemisphere with $F r \simeq 100$ (Fig.3b). This demonstrates that inertia, characterised by the impact Froude number, significantly affects mixing between the impactor and the target.

\subsection{Crater depth scaling}

Before quantifying mixing, we first compare the impacts from our experiments to those from the literature. Fig.5 shows the maximum crater depth in our experiments with $P=0$. When $P$ is fixed, the normalised maximum crater depth $H / R$ depends only on $F r$. We find the following least squares best-fitting power-law

$$
\frac{H}{R}=a_{1} F r^{a_{2}},
$$

where $a_{1}=1.1 \pm 0.05$ and $a_{2}=0.24 \pm 0.01$, as shown as a solid line in Fig.5. Relation (2) is a $\pi$-group scaling law (Melosh, 1989).

To understand the origin of (2), we assume that the kinetic energy of the impactor is converted completely into potential energy in a hemispherical crater of maximum depth $H$, so that

$$
\frac{2}{3} \rho_{i} U^{2} R^{3}=\frac{1}{4} \rho_{t} g H^{4}
$$

where we neglect the density of air compared to $\rho_{i}$ and $\rho_{t}$. Thus, the dimensionless crater depth,

$$
\frac{H}{R}=\left(\frac{8}{3}\right)^{1 / 4}\left(\frac{\rho_{i}}{\rho_{t}}\right)^{1 / 4} F r^{1 / 4}
$$

This energy scaling $H / R \sim F r^{1 / 4}$ is well-known for subsonic impacts of drops with a radius $R<3 \mathrm{~mm}$ in another liquid (Engel, 1967; Pumphrey and Elmore, 1990; Ray et al., 2015; Jain et al., 2019) and subsonic impacts of solid spheres into granular materials (Walsh et al., 2003; Takita and Sumita, 2013; Seaton, 2006).

The experimental exponent $a_{2}=0.24 \pm 0.01$ in (2) agrees well with the $1 / 4$ theoretical prediction given in (4). Even the prefactor $a_{1}=1.1$ is close to the theoretical prediction $(8 / 3)^{1 / 4} \simeq 1.28$ given in (4) for $\rho_{i}=\rho_{t}$. These results confirm that inertia and gravity are the two dominant forces in our experiments, while viscous and surface tension forces play a secondary role. This is the relevant regime for large planetary impacts.

Unlike our experiments, planetary impacts are supersonic. Previous investigations find an exponent $a_{2} \simeq 0.2$ for hypervelocity impacts, suggesting that the $1 / 4$ power-law scaling (4) does not hold (Prieur et al., 2017). Despite this difference in $a_{2}$, the crater depth in our experiments is within $14 \%$ of that predicted by hypervelocity scalings (dotted line in Fig.5 and supplementary section S5). 


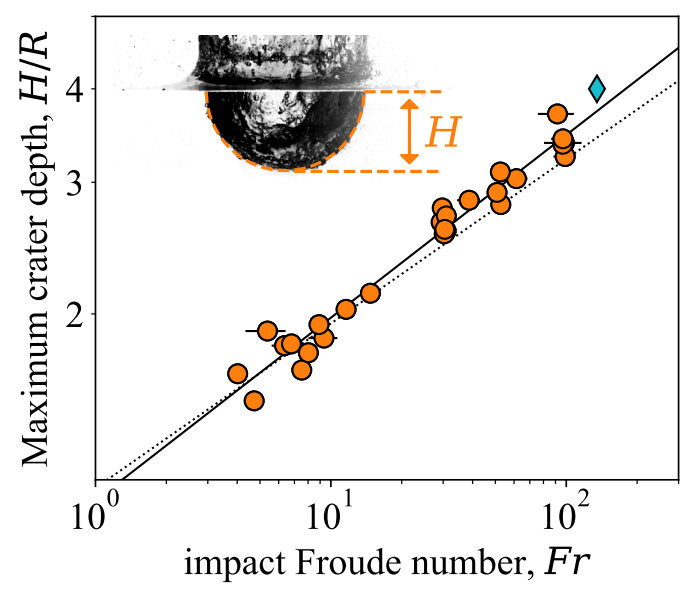

Figure 5: Maximum crater depth $H$ normalised by the impactor radius $R$ as a function of the impact Froude number $F r$. The inset shows the depth $H$ and the crater best-fit semi-circle (dashed yellow curve) in a given experiment; this illustrates that our experimental craters are close to hemispherical. The solid line is the least-square best fit scaling, given by $H / R=a_{1} F r^{a_{2}}$, where $a_{1}=1.1 \pm 0.05$ and $a_{2}=0.24 \pm 0.01$. The dotted line is the scaling law $H / R=1.17 \mathrm{Fr}^{0.22}$ obtained by Schmidt and Housen (1987) in experiments of solid projectiles into water at hypervelocities. We assume a hemispherical crater to convert their scalings for the crater radius into a scaling for the crater depth. In the experiments of Schmidt and Housen (1987), the Froude number ranges from $\mathrm{Fr}=10^{8}$ to $\mathrm{Fr}=3 \times 10^{9}$. The dotted line is therefore an extrapolation over 6 orders of magnitude in $F r$. The blue diamond is the maximum crater depth $H / R \simeq 4$ in an axisymmetric hypervelocity impact simulation of Kendall and Melosh (2016) for $F r \simeq 135$ (see their figure 2b).

\subsection{Mixing scaling}

The mixing between the impactor and the target occurs both during the impact (Fig.2) and within the resultant thermal (Fig.3). Here we determine power-law scalings for mixing, quantifying the dominant processes during both the impact and thermal stage.

At late times, during the thermal stage (Fig.3), the mixing qualitatively agrees with the model of Deguen et al. $(2011,2014)$. This model describes the growth of the thermal as it falls downwards in the magma ocean. It is based on the turbulent entrainment hypothesis, developed by Taylor (1945) and Morton et al. (1956), and inspired by the work of Batchelor (1954). It assumes that the growth rate of the thermal is proportional to its velocity and surface area. This hypothesis implies that the growth of the thermal radius $r$ is linear with the depth $z$ of its centre of mass such that

$$
r=r_{0}+\alpha z
$$

where $\alpha$ is called the entrainment coefficient and $r_{0}$ the effective initial radius of the thermal at $z=0$ (Morton et al., 1956).

As shown in Fig.6a, we recover the linear relation (5) in our experiments, as long as the depth of the centre of mass is larger than about $2 R$. This demonstrates that the turbulent entrainment model (5) accurately describes mixing at long times in our liquid impacts.

The radius $r$ in Fig.6 is based on summations over the entire image (supplementary section S2). Hence, $r$ measures the extent of the structure formed by the thermal and its tail. Accounting for all our experiments, we find that the entrainment coefficient for the thermal with its tail is $\alpha=0.6 \pm 0.1$.

We also measure the half-width of the thermal (Bush et al., 2003), and the radius of the semi-axisymmetric 
a

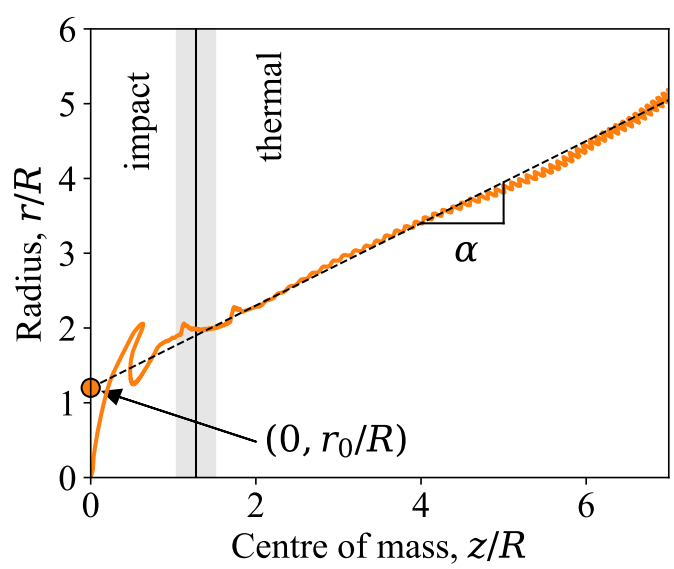

b

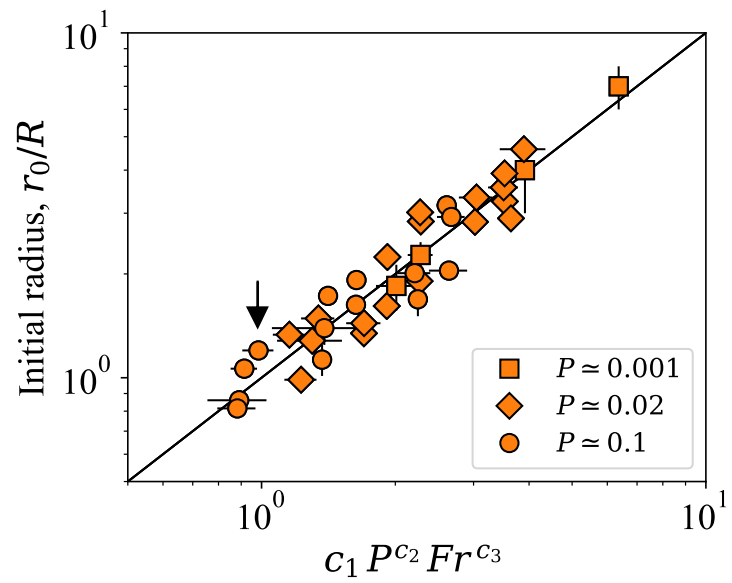

Figure 6: (a) Thermal radius $r$ as a function of the depth of its centre of mass $z$, both normalised by $R$. In this experiment $F r \simeq 8$ and $P \simeq 0.1$. The dashed line is the least-square linear best-fit $r / R=\left(r_{0}+\alpha z\right) / R$, with slope $\alpha=0.55$ and $r_{0} / R \simeq 1.2$. The effective initial radius of the thermal, $r_{0}$, is defined as the value of the best-fit radius $r$ for $z=0$. The arrow denotes the point of coordinates $\left(0, r_{0} / R\right)$. The vertical grey region marks the transition from the early impact to the late thermal stage. (b) Initial radius of the thermal $r_{0}$, normalised by the impactor radius $R$, versus least-squares best-fit power-law scaling as a function of the normalised density excess $P$ and the impact Froude number Fr. Squares: $P \simeq 0.001$; diamonds: $P \simeq 0.02$; circles: $P \simeq 0.1$. The best-fit coefficients are: $c_{1}=0.29 \pm 0.03, c_{2}=-0.19 \pm 0.03, c_{3}=0.4 \pm 0.05$. The arrow locates the experiment shown in (a). Error bars indicate measurement uncertainties.

reconstructed volume (Landeau et al., 2014). Although these quantities depend on an arbitrary binarization threshold, they minimise the effect of the tail. With these definitions, we find an averaged entrainment coefficient of $\alpha=0.25 \pm 0.1$ for the thermal without its tail. This value is indistinguishable from that in pure thermals (Scorer, 1957; Bush et al., 2003).

The large value of $\alpha$ when including the tail is a geometric effect. The vertical extent of the structure is then set by the height of the tail, which nearly equals to the distance $z$ travelled by the thermal. This distance grows faster than the width of the thermal, causing the large $\alpha$ value. Note that the tail is not yet formed at the beginning of the thermal stage, and hence, it does not affect the initial radius $r_{0}$. As the tail contains a small fraction of the impactor mass, we use $\alpha=0.25$ in planetary applications.

Despite the agreement between our experiments and relation (5), an important difference with the model of Deguen et al. $(2011,2014)$ arises. Deguen et al. $(2011,2014)$ assume that $r_{0}$ is equal to the impactor core radius and is independent from the impact velocity. In contrast, we observe that the impact Froude number, and therefore the impact velocity, strongly affects the radius of the thermal at a given depth (Fig.3). To quantify this, in each experiment, we fit relation (5) to the late-time radius to determine the effective initial radius $r_{0}$ of the thermal (Fig.6a). In Fig.6b, we show the dimensionless, effective initial radius, $r_{0} / R$, in all our experiments as a function of the least-square best fit

$$
\frac{r_{0}}{R}=c_{1} P^{c_{2}} \mathrm{Fr}^{c_{3}}
$$

where $F r$ is the impact Froude number and $P$ the normalised excess density. We find $c_{1}=0.29 \pm 0.03$, $c_{2}=-0.19 \pm 0.03, c_{3}=0.4 \pm 0.05$. The sign of the power-law exponent $c_{3}$ agrees with the qualitative 
observation drawn in section 4.1: that the extent of mixing during the impact stage increases with increasing impact Froude number. This trend is intuitive: the larger the impactor kinetic energy, the more mixing the impact produces

The negative sign of the exponent $c_{2}$ is more unexpected. It implies that the larger the excess density, the less mixing occurs at a given depth. As discussed before, the structures growing during crater opening (shown in Fig.4) are related to incompressible Richtmyer-Meshkov or Rayleigh-Taylor instabilities. At a fixed time, mixing by either of these mechanisms increases with the normalised excess density $P$ (Dalziel et al., 1999; Holmes et al., 1999; Lund and Dalziel, 2014). This is at odds with the sign of $c_{2}$, suggesting that the instabilities shown in Fig.4 are not the dominant contribution to mixing.

Some degree of mixing could occur during the rise of the secondary jet (Fig.2d,e and Fig.21,m). In this case, the extent of mixing, and hence $r_{0}$, should scale as the jet size. In previous work, the width and the height of the jet follow the same scaling, which we obtain by equating the gravitational energy in the jet to the kinetic energy of the impactor (Ghabache et al., 2014). With these assumptions, one obtains that the jet size evolves as $F r^{1 / 4}\left(\rho_{i} / \rho_{j e t}\right)^{1 / 4}$, where $\rho_{j e t}$ is the jet mean density. Thus, if mixing occurs during the rise of the jet, we expect $c_{3}=1 / 4$ and $c_{2}>0$. This again cannot explain the observed scaling.

Thus, the only remaining process that can account for scaling (6) is the collapse of the jet, and the subsequent dynamics, illustrated in Fig.2f,g and Fig.2n,o. In section 4.4, we develop a scaling analysis that suggests that jet collapse dominates mixing during giant impacts.

\subsection{Mixing model}

In order to understand mixing between an impactor and a lighter target liquid, we first describe experiments with no excess density, i.e. with $P=0$. In these, after the collapse of the jet, the impacting liquid remains near the surface of the target, but undergoes a slow lateral growth (supplementary Figure S4). This implies that, during the impact stage, the downward momentum of the jet is converted into lateral spreading of the impacting fluid. As the impacting liquid spreads, shear-generated turbulence mixes the impactor with the target. This mixing continues until the kinetic energy is entirely dissipated. In the absence of buoyancy, the impacting liquid never migrates downward as a thermal (supplementary Figure S4).

In contrast, when the impactor is more dense than the target, a buoyancy force arises as the jet releases the impacting liquid. Buoyancy generates a downward momentum. When this balances the momentum of the jet, the impactor stops spreading horizontally and descends as a thermal (Fig.3). For a given impact, the denser the impactor, the earlier it migrates downwards, and hence the smaller the mixing prior to the thermal stage. This rationale explains why the initial extent of the thermal $r_{0}$ decreases as the excess density $P$ increases, as indicated by scaling (6).

We therefore hypothesise that the thermal starts descending when the buoyancy-induced momentum becomes larger than the momentum of the collapsing jet, $M_{j e t}$. A scaling analysis suggests that this takes a 
time

$$
t_{b} \sim \frac{M_{j e t}}{B}
$$

293 where $B=\frac{4}{3} \pi R^{3} P g$ is the total buoyancy of the impacting liquid. The time $t_{b}$ corresponds to the transition 294 from the impact stage (Fig.2) to the thermal stage (Fig.3).

During the time $t_{b}$, the impacting liquid spreads horizontally over a distance

$$
l_{b} \sim \sqrt{M_{j e t}} / B^{1 / 4}
$$

and dimensionless distance,

$$
\tilde{l}_{b}=l_{b} / R \sim P^{-1 / 4} \mathrm{Fr}^{7 / 16} .
$$

Following the turbulent entrainment hypothesis (Morton et al., 1956), we assume that the radius of the dyed region $r_{0}$ scales as the distance travelled, $l_{b}$, times an entrainment coefficient $\alpha$. This implies that the dimensionless volume of the dyed liquid

$$
\tilde{V}=\frac{r_{0}^{3}}{R^{3}} \sim \alpha^{3} P^{-3 / 4} F r^{21 / 16}
$$


a

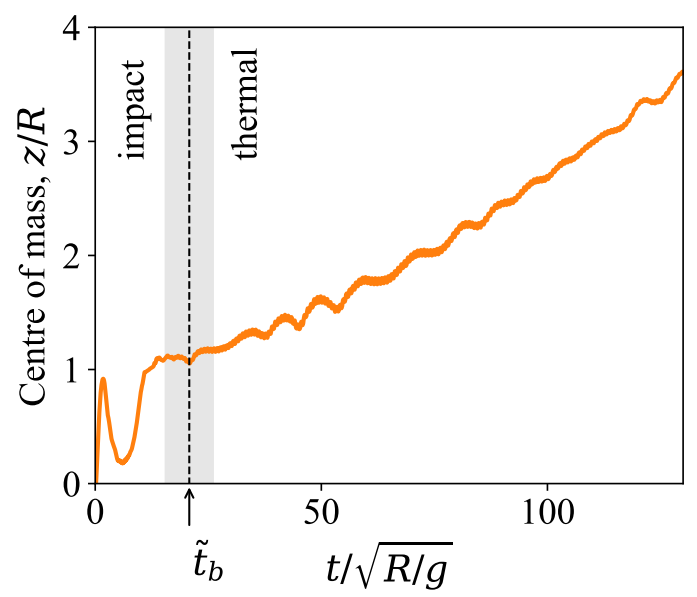

b

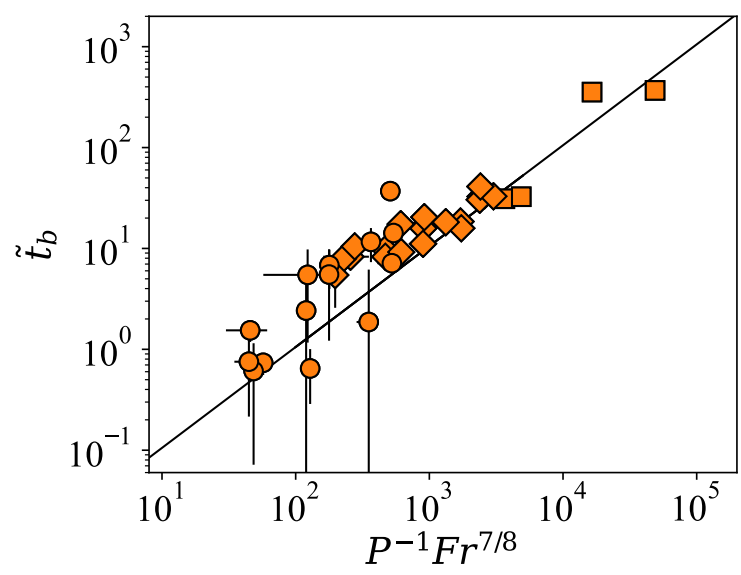

Figure 7: (a) Centre of mass of the dyed fluid, normalised by $R$, as a function of time, normalised by $\sqrt{R / g}$ in a single experiment. The time $\tilde{t}_{b}$ marks the end of the impact stage and the beginning of the thermal stage, in which the centre of mass increases monotonously with time. Oscillations are due to gravity waves on the free surface. (b) Time $\tilde{t}_{b}$, normalised by $\sqrt{R / g}$, measured in all experiments versus its theoretical evolution $P^{-1} F^{7 / 8}$. The solid curve is the least-square best-fit given by $\tilde{t}_{b}=c_{4} P^{-1} F^{7 / 8}$, where $c_{4}=0.010 \pm$ 0.003 is the only fitted coefficient. Squares: $P \simeq 0.001$; diamonds: $P \simeq 0.02$; circles: $P \simeq 0.1$.

Fig.7 shows that the experimentally-measured values of $\tilde{t}_{b}$ agree with the model prediction (10). To test scaling (12), we now estimate the dimensionless thermal volume $\tilde{V}$ (defined in supplementary section S2) at the thermal initiation time $\tilde{t}_{b}$ in all our experiments. As shown in Fig.8, the thermal volume $\tilde{V}$ at $\tilde{t}_{b}$ agrees well with the model prediction from (12). We find the following experimental least-square best-fit

$$
\tilde{V}=(0.019 \pm 0.003) P^{-3 / 4} F r^{21 / 16}
$$

In turbulent shear flows, such as jets or shear layers, the entrainment coefficient is typically $\alpha=0.07-0.37$

(Carazzo et al., 2006; Slessor et al., 2000). This yields a prefactor $\alpha^{3}$ in (12) in the range $0.0003-0.05$, which includes our best-fit prefactor $0.019 \pm 0.003$.

Taking (12) to the power $1 / 3$, we predict $r_{0} / R \sim \alpha P^{-1 / 4} F r^{7 / 16}$, which corresponds to $c_{2}=-1 / 4$ and $c_{3}=7 / 16 \simeq 0.44$. This predicted value of $c_{3}$ is within the error bars of its experimentally-measured value in (6). The predicted value $c_{2}=-1 / 4$ is negative, as the experimental value, but its magnitude is slightly higher than the observation. This may be due to a small mixing contribution from the mushroom-shaped instabilities that grow during crater opening (Fig.4).

\section{Discussion}

\subsection{Comparison with impact simulations}

In the simulations of Kendall and Melosh (2016), the opening of a crater stretches the impactor into a lenticular volume. This also occurs in our experiments. However, we observe that the impactor spreads over the entire crater floor (Fig. 2k), and not within an inner crater as in Kendall and Melosh (2016). 


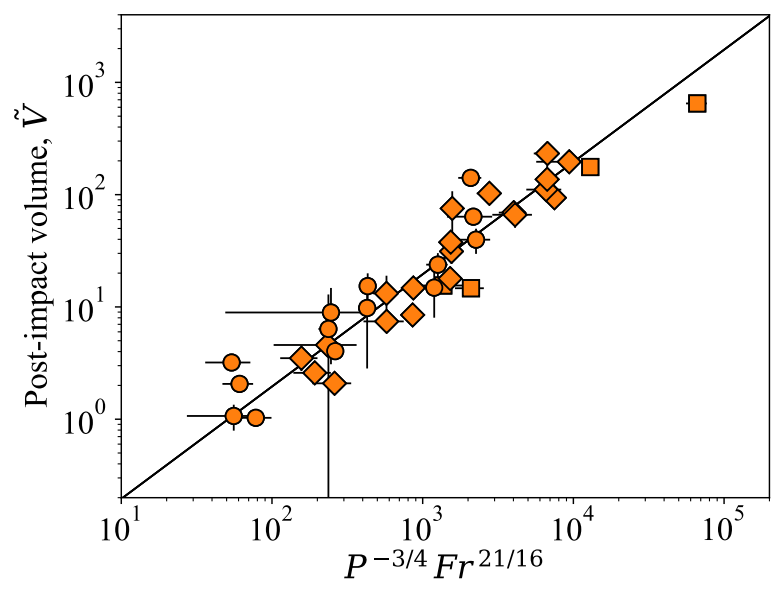

Figure 8: Measured post-impact volume of dyed fluid, normalised by the impactor volume, versus its theoretical evolution $P^{-3 / 4} \mathrm{Fr}^{21 / 16}$. Squares: $P \simeq 0.001$; diamonds: $P \simeq 0.02$; circles: $P \simeq 0.1$. Error bars indicate measurement uncertainties. In this figure, the volume of dyed fluid $\tilde{V}$ is equal to $\left(3 s_{x}\right)^{2} 3 s_{z}$, where $s_{x}$ and $s_{z}$ are the second central moments of the concentration $c(x, z)$ in the horizontal and vertical direction, respectively (supplementary section S2). The volume $\tilde{V}$ is measured at time $\tilde{t}_{b}$, as defined in Fig.7a, marking the beginning of the buoyancy-driven stage shown in Fig. 3. The solid curve is the least-square best-fit given by $\tilde{V}=c_{5} P^{-3 / 4} \mathrm{Fr}^{21 / 16}$, where $c_{5}=0.019 \pm 0.003$ is the only fitted coefficient.

In experiments, the impactor is always entrained by the central jet (Fig. 2m). This happens in the 3D simulations of Kendall and Melosh (2016), but not in their 2D simulations.

In the vertical impact of Kendall and Melosh (2016), at impact speed $U=11.5 \mathrm{~km} \mathrm{~s}^{-1}$, the maximum crater depth is $\simeq 400 \mathrm{~km}$, i.e. $H \simeq 4 R$. In this simulation, $F r \simeq 135$ and $P=\left(\rho_{i}-\rho_{t}\right) / \rho_{t} \simeq 0.14$, where $\rho_{i}$ is the mean density of the impactor. This definition for $P$ is supported by our results, which suggest that mixing depends only on the jet momentum and the total buoyancy of the impactor (section 4.4). With this Froude number, our scaling (2) predicts $H \simeq 3.7 R$, which deviates by only $7.5 \%$ from the numerical value (Fig. 5). Incorporating the factor $\left(\rho_{i} / \rho_{t}\right)^{1 / 4}$ from (4) into scaling (2), we predict $H \simeq 3.9 R$, which now deviates by only $3 \%$.

With $P \simeq 0.14$ and $F r \simeq 135$, our scaling (13) predicts a post-impact volume $\tilde{V} \simeq 51$ at time $t_{b} \simeq$ $500 \pm 200 \mathrm{~s}$. This means that the impactor core is dispersed within a volume 51 times the impactor. In figure A.2 of Kendall and Melosh (2016), at a comparable time of $500 \mathrm{~s}$, the vertical extent $l_{z} \simeq 1600 \mathrm{~km}$ and horizontal extent $l_{x} \simeq 200 \mathrm{~km}$. Assuming a cylindrical structure, this leads to $\tilde{V} \simeq 3\left(l_{x}^{2} l_{z}\right) / 16 R^{3} \simeq 12$. At a slightly later time of $1000 \mathrm{~s}, l_{z} \simeq 800 \mathrm{~km}$ and $l_{x} \simeq 400 \mathrm{~km}$ (figure 6 of Kendall and Melosh, 2016), leading to $\tilde{V} \simeq 3\left(l_{x}^{2} l_{z}\right) / 16 R^{3} \simeq 24$. These values for $\tilde{V}$ are 2 to 4 times smaller than that predicted by our scaling law, but they agree reasonably well when considering that $\tilde{V}$ varies over 3 orders of magnitude in our experiments (Fig.8). Furthermore, this $50 \%-75 \%$ discrepancy in volume is only a $20 \%$ - 38\% discrepancy in radius.

These differences in mixing may come from compressibility, which is negligible in our experiments. In simulations, compressibility effects are significant at the beginning of the opening of the crater. A fraction of the impactor energy is then transported away by shock waves, and hence is not available for mixing. Compressibility effects may be responsible for the inner crater, which limits the spreading of the impactor 
core in the simulations of Kendall and Melosh (2016) (see their figure 2). At later times, as the crater expands, the impact kinetic energy is converted into potential energy and imparted to an increasing volume of target material. Thus, flow velocities rapidly decrease to subsonic speeds. In the supplementary movies of Kendall and Melosh (2016), the velocity of the metal is $\lesssim 300 \mathrm{~m} \mathrm{~s}^{-1}$ after the crater reaches its maximum size. Such speeds correspond to a low Mach number $\sim 0.05-0.1$. Compressibility is therefore unlikely to affect mixing after the cratering stage. Assuming compressibility is responsible for the differences in mixing, one needs to correct our predictions by a factor of 0.25 to 0.5 in $\tilde{V}$, and hence 0.6 to 0.8 in $r_{0}$.

The lower level of mixing in simulations might also originate from a lower level of turbulence. Our predictions would then need no correction factor. Despite the high resolution used by Kendall and Melosh (2016), the grid size, which ranges from 2.5 to $10 \mathrm{~km}$, is much larger than the smallest turbulent scale $R R e^{-3 / 4} \lesssim 0.1 \mathrm{~mm}$ (caption in supplementary Table 1 ). This explains why the mixing increases with decreasing grid size in simulations (supplementary figures A1 and A3 in Kendall and Melosh, 2016). For example, the post-impact volume $\tilde{V}$ at time $500 \mathrm{~s}$ is multiplied by $\sim 2$ when decreasing the grid size from 5 to $2.5 \mathrm{~km}$. From these observations, Kendall \& Melosh predict a larger level of mixing when reaching a more turbulent regime. This agrees with our experimental scalings.

Finally, our experiments model the impactor as a single phase, while it is differentiated into a core and a mantle in the simulations of Kendall and Melosh (2016). In supplementary section S6, we show that the jet likely entrains the core of a differentiated impactor during crater collapse. With this assumption, mixing depends only on the total buoyancy and momentum of the collapsing jet, as inferred in section 4.4. These quantities do not depend on the mass distribution in the impactor. We therefore expect our scaling laws to hold for differentiated impactors.

\subsection{Implications for metal-silicate mixing}

In our experiments, we observe two main stages. At early times, the impact controls the dynamics. The impactor opens a crater, which collapses into an upward jet (Fig.2); the jet stops and collapses due to gravity, releasing the denser impacting liquid. At late times, the impacting liquid descends into the target as a turbulent thermal (Fig.3). The late thermal stage is similar to that observed in previous investigations that neglect the impact process (Deguen et al., 2011, 2014).

However, Deguen et al. $(2011,2014)$ assumed that the initial thermal volume is equal to the volume of the impactor core and is independent of the impact velocity. In contrast, we find that increasing the impact velocity strongly increases the initial thermal volume (Fig.3). We found that the volume of mixed target, which is an analogue for the equilibrated silicates, increases with increasing impact Froude number $F r=U^{2} / g R$ (Fig.6 and Fig.8, and scalings (6) and (13)). We now translate these results into parameters that can be used in geochemical models of Earth's accretion.

Applied to planetary impacts, the impact Froude number is the ratio of the kinetic energy to the gravita- 
tional potential energy at impact, given by

$$
F r=\frac{U^{2}}{R G\left(M+M_{t}\right) /\left(R+R_{t}\right)^{2}}=2\left(1+\frac{R_{t}}{R}\right) \frac{U^{2}}{U_{e}^{2}},
$$

where $U$ is the impact velocity, $U_{e}=\sqrt{2 G\left(M+M_{t}\right) /\left(R+R_{t}\right)}$ the mutual escape velocity, $G$ the gravitational constant, $R$ and $R_{t}$ are the impactor and target radius, and $M$ and $M_{t}$, the mass of the impactor and target planet, respectively. During Earth's accretion, the impact velocity is usually one to three times the escape velocity (Agnor et al., 1999). Thus, the impact Froude number scales as $\left(1+R_{t} / R\right)$. This number is on the order of unity for a giant impact, but it is much larger than unity for a small impactor of less than about 100 $\mathrm{km}$ in radius. Since mixing increases with the Froude number (Fig.8), this implies that small, low-energy impacts generate much more mixing, relative to their size, than giant impacts.

To quantify this dependence of mixing on the impactor size, we now derive the amount of silicates entrained in the thermal. Our experimental scaling (6) for the initial volume of the thermal relates the initial thermal radius, $r_{0}$, to the impact Froude number, $F r$, and the dimensionless excess density, $P$. Using expression (14) for $F r$, this scaling then becomes

$$
\frac{r_{0}}{R}=c_{1} 2^{c_{3}} f_{m}^{c_{2}}\left(\frac{\rho_{m}-\rho_{s}}{\rho_{s}}\right)^{c_{2}}\left(\frac{U}{U_{e}}\right)^{2 c_{3}}\left(1+\frac{R_{t}}{R}\right)^{c_{3}},
$$

where $c_{1}=0.29 \pm 0.03, c_{2}=-0.19 \pm 0.03, c_{3}=0.4 \pm 0.05$ are experimental constants, $\rho_{m}$ the density of metal, $\rho_{s}$ the density of silicates and $f_{m}$ the initial volume fraction of metal in the impactor. We define $P=$ $\left(\bar{\rho}-\rho_{s}\right) / \rho_{s}$, as the normalised difference between the mean density of the impactor $\bar{\rho}=f_{m} \rho_{m}+\left(1-f_{m}\right) \rho_{s}$ and the silicate density.

Following the results of Deguen et al. (2014), we assume that the silicates entrained in the thermal efficiently equilibrate with the impactor metal. We define the dimensionless mass of equilibrated silicates $\Delta=M_{s} / M_{m}$, where $M_{s}$ is the mass of entrained silicates, and $M_{m}$ the impactor core mass. In the aftermath of the impact, just before the thermal descends into the magma ocean, $\Delta$ takes the value

$$
\Delta_{0}=\frac{\rho_{s}}{\rho_{m}}\left[\frac{1}{f_{m}}\left(\frac{r_{0}}{R}\right)^{3}-1\right]
$$

where, as before, $r_{0}$ is the effective initial radius of the thermal. Inserting scaling (15) for $r_{0}$ into (16), we compute the normalised mass of silicates $\Delta_{0}$, mixed with metal by the impact stage, prior to its descent in the magma ocean, as a function of the impact velocity, the impactor size and its volumetric metal fraction.

Fig.9a shows $\Delta_{0}$ as a function of the impactor size. The mass of mixed silicates monotonically increases with the impact velocity and the radius of the target relative to that of the impactor. For a small impact with a $100 \mathrm{~km}$-sized body at escape velocity, the impactor core mixes with 168 times its mass (circle in Fig.9a). In contrast, a giant impact with a Mars-sized impactor, similar to the canonical Moon-forming scenario 
(Canup, 2004), generates little mixing with a mass of mixed silicates less than 1.5 times the impactor core (diamond in Fig.9a).

Fig.9a illustrates that mixing by giant impacts is highly sensitive to the impact speed and impactor size. Another Moon-forming scenario is the impact of a high-speed sub-Mars object on a fast-spinning Earth (Ćuk and Stewart, 2012). For this scenario, we predict that the impactor core mixes with 12 times its mass of silicates (square in Fig.9a), more than 8 times the mass predicted for the canonical scenario (diamond). This is counter-intuitive as the total kinetic energy of the impactor in Ćuk and Stewart (2012)'s scenario is 5 times less than in the canonical scenario. This scenario involves a smaller impactor at higher speed, which therefore corresponds to a larger impact Froude number according to (14), forcing more efficient mixing.

In the previous paragraphs, we discussed the efficiency of mixing by the impact stage, when the metal is at the top of the magma ocean. However, geochemical data record the end result of mixing, when the thermal reaches the bottom of the ocean. To predict this final mixing, we use the effective initial radius $r_{0}$ of the thermal in the aftermath of an impact, as predicted by our scaling (15), as the new initial condition for the growth of the thermal radius in the ocean (Deguen et al., 2011)

$$
r=r_{0}+\alpha z
$$

where $z$ the depth in the magma ocean and $\alpha$ the entrainment coefficient. The mass of equilibrated silicates at the bottom of the magma ocean, normalised by the impactor core mass, is then

$$
\Delta=\frac{\rho_{s}}{\rho_{m}}\left[\frac{1}{f_{m}}\left(\frac{r_{0}}{R}+\alpha \frac{z_{m o}}{R}\right)^{3}-1\right]
$$

where $k$ is the fraction of equilibrated metal, $D$ is the metal-silicate partition coefficient. With this definition,

where $z_{m o}$ is the total depth of the magma ocean. We use (15) and (18) to estimate $\Delta$ for a given impact, knowing the impact velocity, the impactor size and the metal fraction of the impactor.

Fig.9b shows the mass of mixed silicates predicted by (18), assuming that the magma ocean is $30 \%$ of the depth of the mantle. At the base of the magma ocean, we again predict more efficient mixing for small impacts (circle) than for giant impacts (diamond and square). The three curves indicate a strong effect of the impact speed. Fig.9c shows that the total mass of mixed silicates, when we include the mixing by the impact process, is up to 100 times larger than the previous estimates of Deguen et al. $(2011,2014)$.

We define the degree of chemical equilibration, $\mathcal{E}$, for a given chemical element, as the mass exchanged between metal and silicates to the maximum exchanged mass if the metal equilibrates with an infinite volume of silicates. From mass conservation equations, Deguen et al. (2014) showed that

$$
\mathcal{E}=\frac{k}{1+D / \Delta}
$$



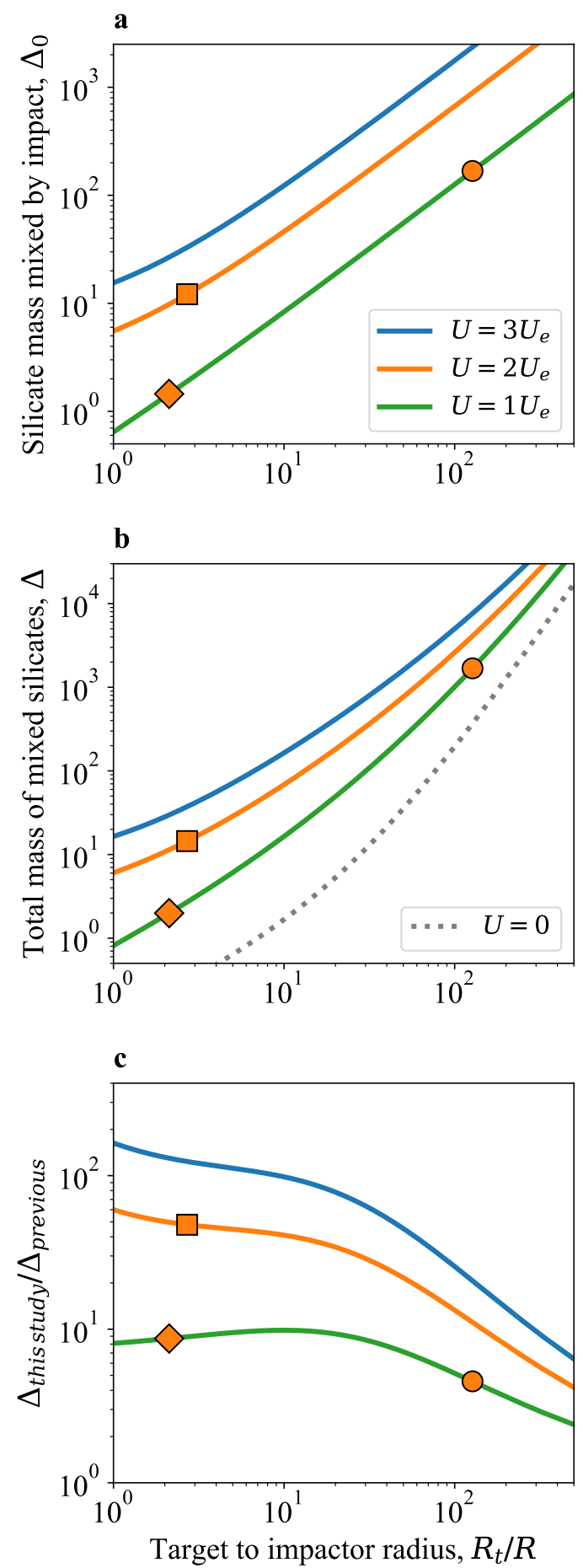

Figure 9: Mass of silicates mixed with metal by the impact stage $\Delta_{0}$, prior to the descent into the magma ocean (a), and by the impact and thermal stage at the bottom of the magma ocean $\Delta(\mathrm{b})$, as a function of the target to impactor radius $R_{t} / R$. The silicate mass is normalised by the mass of impactor core. (c) Total mass of mixed silicates $\Delta$ at the bottom of the magma ocean as predicted by our scalings that include the impact stage, divided by $\Delta$ computed from the model of Deguen et al. (2014), as a function of the target to impactor radius $R_{t} / R$. Green: impact velocity $U$ equals to the mutual escape velocity $U_{e}$. Orange: $U=2 U_{e}$. Blue: $U=3 U_{e}$. Grey dotted: model of Deguen et al. (2014) with no impact stage. Circle: impactor of $100 \mathrm{~km}$ in radius onto an Earth-sized target. Diamond: canonical Moon-forming scenario with a Mars-sized impactor (Canup, 2004). Square: Moon-forming scenario with an impactor mass 20 times smaller than the target onto a fast-spinning Earth (Ćuk and Stewart, 2012). We use relations (16) and (15) to compute $\Delta_{0}$ and $\Delta$, taking $f_{m}=0.16,\left(\rho_{m}-\rho_{s}\right) / \rho_{s}=1, \alpha=0.25$ and assuming the magma ocean depth is $30 \%$ the mantle depth. 

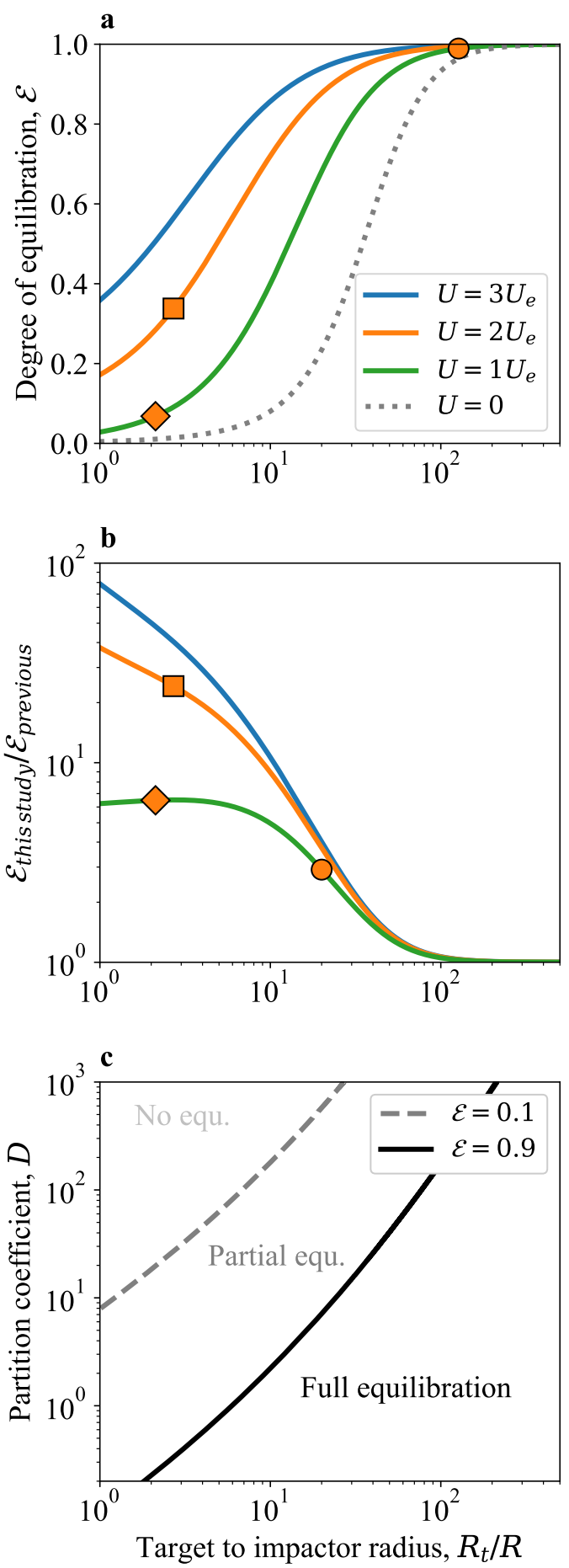

Figure 10: (a) Degree of equilibration $\mathcal{E}$ at the bottom of the magma ocean as a function of the target to impactor radius $R_{t} / R$ using relations (18) and (19), and taking a partition coefficient of 30 and $\alpha=0.25$. Green: $U=U_{e}$. Orange: $U=2 U_{e}$. Blue: $U=3 U_{e}$. Grey dotted: model of Deguen et al. (2014) with no impact stage. Circle: impactor of $100 \mathrm{~km}$ in radius onto an Earth-sized target at $U=U_{e}$. Diamond: canonical Moon-forming scenario with a Mars-sized impactor (Canup, 2004). Square: Moon-forming scenario of Ćuk and Stewart (2012). (b) Ratio of the degree of equilibration $\mathcal{E}$ predicted by our scalings that include the impact stage to the degree of equilibration predicted by the model of Deguen et al. (2014). (c) Regime diagram for metal-silicate equilibration as a function of the target to impactor radius $R_{t} / R$ and the partition coefficient $D$, with $U=U_{e}$. For all panels, we take $f_{m}=0.16$, $\left(\rho_{m}-\rho_{s}\right) / \rho_{s}=1$, $k=1$ and we assume that the depth of the magma ocean is $40 \%$ that of the mantle (Siebert et al., 2012; Fischer et al., 2015).

et al., 2010).

Fig.10a illustrates our prediction for the degree of equilibration when using (15) and (18). We assume

${ }_{411} k=1$, as suggested by Deguen et al. (2014), and choose $D=30$. Fig.10a shows that we expect full 
equilibration for small impactors with radii less than about 1/30 that of the target. For an Earth-sized target, this corresponds to impactors smaller than about $200 \mathrm{~km}$. In contrast, the extent of equilibration is highly variable and very sensitive to the impact speed for giant collisions. For the Mars-sized impactor of the canonical Moon-forming scenario (diamond), the degree of equilibration $\mathcal{E}$ is 0.07 while it is 0.34 for the Mercury-sized impactor proposed by Ćuk and Stewart (2012) (square).

As shown in Fig.10b, the degree of equilibration predicted by our scalings is up to 20 times larger than previous estimates that neglect the effect of the impact speed. We therefore anticipate that our new scalings for metal-silicate mixing will strongly affect geochemical models of Earth's formation (Rubie et al., 2015; Badro et al., 2018). They will also affect the timing of core formation, which is deduced from extinct radioactivity (Rudge et al., 2010). To illustrate this, we consider impactors at escape velocity with a radius 30 times smaller than that of the proto-Earth. Our scalings predict that the mean degree of equilibration increases from 0.4 to 0.8 for these impacts (Fig.10ab). According to Figure 3b of Rudge et al. (2010), the duration of core formation from the Hf-W systematics is about $30 \mathrm{Myr}$ for an equilibration $\mathcal{E}=0.8$, but it is hardly constrained for $\mathcal{E}=0.4$ and could range from fifty to several hundred million years.

Similarly, the depth of the magma ocean deduced from siderophile elements depends on the assumed degree of equilibration. For impactors with a radius 20 times smaller than the target, the value of $\mathcal{E}$, which was close to 0.2 with previous models, is now in the range $0.7-0.95$ (Fig.10ab). With such an increase, figure 7 from Fischer et al. (2017) predicts that the depth of the magma ocean decreases by up to $43 \%$.

By definition, the degree of equilibration depends on the partition coefficient between metal and silicates, and therefore on the chemical element. This is illustrated in Fig.10c. For a metal-silicate partition coefficient $D \lesssim 0.2$, we predict full equilibration with $\mathcal{E}>0.9$ for most impacts, except for giant impactors larger than half the target in radius. In contrast, for $D=100$, partial equilibration is the norm.

In conclusion, we found that mixing primarily depends on the impact Froude number, which measures the importance of the impactor kinetic energy to its gravitational energy. Applied to Earth's formation, our scalings relate the mass of silicates entrained with metal as a function of the impact velocity, the impactor size and the depth of the magma ocean. We predict that the impact stage is responsible for up to $94 \%$ of the total mixing at the base of the magma ocean. This impact-induced mixing was entirely neglected in previous studies. Its effects on chemical transfers and geochemical models of core formation therefore deserves future investigations.

\section{Acknowledgements}

We are grateful to the late H. Jay Melosh and an anonymous reviewer for their valuable comments that significantly improved this manuscript. We thank Peter Olson, Miki Nakajima, Hélène de Maleprade, Paul Jarvis, Amy Bonsor, Kai Wünnemann and Merlin Etzold for fruitful discussions at key moments during this project. We are extremely grateful to David Page-Croft, Colin Hitch, Jamie Partridge, John Milton, and 
Mark Hallworth for their valuable help in setting up the experimental and visualization techniques. This project has received funding from the European Union's Horizon 2020 research and innovation program under the Marie Sklodowska-Curie Grant Agreement 703767. This work was also supported by the Programme National de Planétologie (PNP) of CNRS-INSU co-funded by CNES. RD and VL have received funding from the European Research Council (ERC) under the European Unions Horizon 2020 research and innovation programme (grant agreement 716429). This study contributes to the IdEx Université de Paris ANR-18-IDEX-0001.

\section{Author contributions}

ML, RD, DP, JN, SD contributed to the conception of the laboratory experiments and the measurement techniques. ML and DP conducted the experiments and analysed the data. All co-authors participated to the interpretation of data. ML wrote the paper; all co-authors carefully revised the manuscript and approved the final version.

\section{Declaration of interests}

The authors declare that they have no known competing financial interests or personal relationships that could have appeared to influence the work reported in this paper.

\section{References}

Agnor, C. B., Canup, R. M., Levison, H. F., 1999. On the character and consequences of large impacts in the late stage of terrestrial planet formation. Icarus 142 (1), 219-237.

Badro, J., Aubert, J., Hirose, K., Nomura, R., Blanchard, I., Borensztajn, S., Siebert, J., 2018. Magnesium partitioning between earth's mantle and core and its potential to drive an early exsolution geodynamo. Geophys. Res. Lett. 45 (24), 13-240.

Batchelor, G. K., 1954. Heat convection and buyoancy effects in fluids. Quart. J. Roy. Met. Soc. 80 (345), 339-358.

Bercovici, D., Ricard, Y., 2014. Plate tectonics, damage and inheritance. Nature 508 (7497), 513-516.

Bisighini, A., Cossali, G., Tropea, C., Roisman, I., 2010. Crater evolution after the impact of a drop onto a semi-infinite liquid target. Physical Review E 82 (3), 036319.

Blau, P., Axon, H., Goldstein, J., 1973. Investigation of the canyon diablo metallic spheroids and their relationship to the breakup of the canyon diablo meteorite. J. Geophys. Res. 78 (2), 363-374.

Bush, J. W. M., Thurber, B. A., Blanchette, F., 2003. Particle clouds in homogeneous and stratified environments. J. Fluid Mech. 489, $29-54$.

Canup, R. M., 2004. Simulations of a late lunar-forming impact. Icarus 168 (2), 433-456.

Carazzo, G., Kaminski, E., Tait, S., 2006. The route to self-similarity in turbulent jets and plumes. J. Fluid Mech. 547, 137.

Cenedese, C., Dalziel, S. B., 1998. Concentration and depth fields determined by the light transmitted through a dyed solution. Proceedings of the 8th International Symposium on FlowVisualization 8, 1-37.

Chambers, J. E., 2004. Planetary accretion in the inner Solar System. Earth Planet. Sci. Lett. 223 (3-4), 241-252.

Ćuk, M., Stewart, S., 2012. Making the moon from a fast-spinning earth: a giant impact followed by resonant despinning. Science 338 (6110), 1047-1052. 
Dahl, T. W., Stevenson, D. J., 2010. Turbulent mixing of metal and silicate during planet accretion - And interpretation of the Hf-W chronometer. Earth Plan. Sci. Lett. 295 (1-2), 177-186.

Dalziel, S. B., Linden, P. F., Youngs, D. L., 1999. Self-similarity and internal structure of turbulence induced by Rayleigh-Taylor instability. J. Fluid Mech. 399, 1-48.

Deguen, R., Landeau, M., Olson, P., 2014. Turbulent metal-silicate mixing, fragmentation, and equilibration in magma oceans. Earth Planet. Sci. Lett. 391, 274-287.

Deguen, R., Olson, P., Cardin, P., 2011. Experiments on turbulent metal-silicate mixing in a magma ocean. Earth and Planetary Science Letters $310(3-4), 303-313$.

Ellison, T., Turner, J., 1959. Turbulent entrainment in stratified flows. J. Fluid Mech. 6 (03), 423-448.

Engel, O., 1967. Initial pressure, initial flow velocity, and the time dependence of crater depth in fluid impacts. Journal of applied physics 38 (10), 3935-3940.

Fischer, R., Campbell, A., Ciesla, F., 2017. Sensitivities of earth's core and mantle compositions to accretion and differentiation processes. Earth Planet. Sci. Lett. 458, 252-262.

Fischer, R., Nakajima, Y., Campbell, A., Frost, D., Harries, D., Langenhorst, F., Miyajima, N., Pollok, K., Rubie, D., 2015. High pressure metal-silicate partitioning of Ni, Co, V, Cr, Si, and O. Geochem. Cosmochim. Acta 167, 177-194.

Ghabache, É., Séon, T., Antkowiak, A., 2014. Liquid jet eruption from hollow relaxation. J. Fluid Mech. 761, $206-219$.

Holmes, R., Dimonte, G., Fryxell, B., Gittings, M., Grove, J., Schneider, M., Sharp, D., Velikovich, A., Weaver, R., Zhang, Q., 1999. Richtmyer-Meshkov instability growth: experiment, simulation and theory. J. Fluid Mech. 389, 55-79.

Ichikawa, H., Labrosse, S., Kurita, K., 2010. Direct numerical simulation of an iron rain in the magma ocean. J. Geophys. Res. 115, doi:10.1029/2009JB006427.

Jacobs, J., Sheeley, J., 1996. Experimental study of incompressible richtmyer-meshkov instability. Physics of Fluids 8 (2), $405-415$.

Jain, U., Jalaal, M., Lohse, D., Van Der Meer, D., 2019. Deep pool water-impacts of viscous oil droplets. Soft matter 15, 4629-4638.

Kendall, J., Melosh, H., 2016. Differentiated planetesimal impacts into a terrestrial magma ocean: Fate of the iron core. Earth Planet. Sci. Lett. 448, 24-33.

Kleine, T., Munker, C., Mezger, K., Palme, H., 2002. Rapid accretion and early core formation on asteroids and the terrestrial planets from Hf-W chronometry. Nature 418 (6901), 952-955.

Landeau, M., Deguen, R., Olson, P., 2014. Experiments on the fragmentation of a buoyant liquid volume in another liquid. J. Fluid Mech. 749, 478-518.

Lherm, V., Deguen, R., 2018. Small-scale metal/silicate equilibration during core formation: The influence of stretching enhanced diffusion on mixing. J. Geophys. Res. 123 (12), 10-496.

Lund, H. M., Dalziel, S. B., 2014. Bursting water balloons. J. Fluid Mech. 756, 771-815.

Maas, C., Manske, L., Wünnemann, K., Hansen, U., 2021. On the fate of impact-delivered metal in a terrestrial magma ocean. Earth Plan. Sci. Lett. 554, doi: 10.1016/j.eps1.2020.116680.

Melosh, H. J., 1990. Giant impacts and the thermal state of the early earth. In: Newsom, H., Jones, J. (Eds.), Origin of the Earth. Oxford University Press Inc.

Melosh, J., 1989. Impact Cratering: A Geologic Process. Oxford University Press Inc.

Morton, B. R., 1959. Forced plumes. J. Fluid Mech. 5 (1), 151-163.

Morton, B. R., Taylor, G., Turner, J. S., 1956. Turbulent gravitational convection from maintained and instantaneous sources. Proc. R. Soc. Lond. 234 (1196), 1-23.

Nakajima, M., Golabek, G., Wünnemann, K., Rubie, D., Burger, C., Melosh, H. J., Jacobson, S., Manske, L., Hull, S., 2020. Scaling laws for the geometry of an impact-induced magma ocean. arXiv, submitted to Earth Planet. Sci. Lett., 2004.04269.

Olson, P., Deguen, R., Rudolph, M., Zhong, S., 2015. Core evolution driven by mantle global circulation. Phys. Earth Planet. Int. 243, $44-55$.

Patterson, C., Tilton, G., Inghram, M., 1955. Age of the earth. Science 121 (3134), 69-75. 
Prieur, N., Rolf, T., Luther, R., Wünnemann, K., Xiao, Z., Werner, S., 2017. The effect of target properties on transient crater scaling for simple craters. J. Geophys. Res.-Planets 122 (8), 1704-1726.

Pumphrey, H., Elmore, P., 1990. The entrainment of bubbles by drop impacts. J. Fluid Mech. 220, 539-567.

Qaddah, B., Monteux, J., Clesi, V., Bouhifd, M., Le Bars, M., 2019. Dynamics and stability of an iron drop falling in a magma ocean. Phys. Earth Planet. Int. 289, 75-89.

Ray, B., Biswas, G., Sharma, A., 2015. Regimes during liquid drop impact on a liquid pool. J. Fluid Mech. 768, $492-523$.

Rayleigh, L., 1883. Investigation of the character of the equilibrium of an incompressible heavy fluid of variable density. Proc. Lond. Math. Soc. 14, 70-177.

Ricard, Y., Sramek, O., Dubuffet, F., 2009. A multi-phase model of runaway core-mantle segregation in planetary embryos. Earth Planet. Sci. Lett. 284 (1-2), 144-150.

Rubie, D., Jacobson, S., Morbidelli, A., O’Brien, D., Young, E., de Vries, J., Nimmo, F., Palme, H., Frost, D., 2015. Accretion and differentiation of the terrestrial planets with implications for the compositions of early-formed solar system bodies and accretion of water. Icarus 248, 89-108.

Rubie, D. C., Melosh, H. J., Reid, J. E., Liebske, C., Righter, K., 2003. Mechanisms of metal-silicate equilibration in the terrestrial magma ocean. Earth Planet. Sci. Lett. 205 (3-4), 239-255.

Rudge, J. F., Kleine, T., Bourdon, B., 2010. Broad bounds on Earth's accretion and core formation constrained by geochemical models. Nat. Geosci. 3 (6), 439-443.

Samuel, H., 2012. A re-evaluation of metal diapir breakup and equilibration in terrestrial magma oceans. Earth Planet. Sci. Lett. 313, $105-114$.

Schmidt, R., Housen, K., 1987. Some recent advances in the scaling of impact and explosion cratering. International Journal of Impact Engineering 5 (1-4), 543-560.

Scorer, R. S., 1957. Experiments on convection of isolated masses of buoyant fluid. J. Fluid Mech. 2 (6), $583-594$.

Seaton, M., 2006. Phd thesis: Particle resuspension by liquid droplets. DAMTP, University of Cambridge.

Siebert, J., Badro, J., Antonangeli, D., Ryerson, F., 2012. Metal-silicate partitioning of ni and co in a deep magma ocean. Earth and Planet. Sci. Lett. 321-322, 189-197.

Slessor, M., Zhuang, M., Dimotakis, P., 2000. Turbulent shear-layer mixing: growth-rate compressibility scaling. J. Fluid Mech. 414, $35-45$.

Takita, H., Sumita, I., 2013. Low-velocity impact cratering experiments in a wet sand target. Physical Review E 88 (2), 022203.

Taylor, G. I., 1945. Dynamics of a hot mass rising in air. U.S. Atomic Energy Commission, MDDC-919, LADC-276.

Tonks, W. B., Melosh, H. J., 1993. Magma ocean formation due to giant impacts. J. Geophys. Res. 98 (E3), $5319-5333$.

Ulvrová, M., Coltice, N., Ricard, Y., Labrosse, S., Dubuffet, F., Velímskỳ, J., Srámek, O., 2011. Compositional and thermal equilibration of particles, drops, and diapirs in geophysical flows. Geochem. Geophy. Geosystems. 12 (10).

Vdovykin, G., 1973. The canyon diablo meteorite. Space Science Reviews 14 (6), 758-831.

Wacheul, J.-B., Le Bars, M., 2018. Experiments on fragmentation and thermo-chemical exchanges during planetary core formation. Phys. Earth Planet. Int. 276, 134-144.

Walsh, A., Holloway, K., Habdas, P., de Bruyn, J., 2003. Morphology and scaling of impact craters in granular media. Physical review letters 91 (10), 104301.

Worthington, A. M., 1908. A study of splashes. Longmans, Green, and Company. 